\title{
Parvalbumin neurons as a hub in autism spectrum disorders
}

Autism spectrum disorder (ASD) is a heterogeneous and complex group of neurodevelopmental disorders characterized by impairments in social behavior and language/communication and the presence of repetitive or stereotyped patterns of behaviors. Up to 1 in 70 children (with a 4:1 preference in boys) is diagnosed with ASD before the age of three, however no effective treatment directed at the core symptoms of ASD is available on the market. A major challenge in ASD research remains the elucidation of the disease etiology. It is widely accepted that a combination of genetic, epigenetic, and environmental risk factors contribute to the development of ASD, yet with several hundreds (!) of risk genes, no consistent theory concerning ASD pathophysiology has been established (de la Torre-Ubieta, Won, Stein, \& Geschwind, 2016). It is assumed that these risk factors cause (directly or indirectly) impairments in synaptic transmission, resulting in regionspecific and occasionally temporally restricted imbalances between excitation and inhibition in the brain, ultimately leading to behavioral and cognitive deficits. Recently, an unconventional but interesting hypothesis was put forward, suggesting that ASD is not a disorder, but rather the end result of functional adaptive/homeostatic mechanisms during neurodevelopment under pre-stressed conditions. According to this hypothesis, ASD is not even a disease, adding another dimension on explanative theories on ASD etiology.

In order to facilitate treatment approaches, identification of convergent pathways, or common endpoints, during ASD development are highly needed. In our research projects, the focus is centered on a protein called parvalbumin (PV). PV is a calcium-binding protein expressed in a subset of GABAergic interneurons in the brain. Several studies have shown that signaling is impaired in PV-expressing interneurons (hereafter termed Pvalb neurons) and/or that their numbers are decreased in human ASD patients and different ASD mouse models (Hashemi, Ariza, Rogers, Noctor, \& Martínez-Cerdeño; Wöhr et al., 2015). Moreover, knockout mice for PV (PV-/-) show a strong ASD-associated phenotype at the juvenile stage ( 1 month of age). Of note, the human PVALB gene is not found on the list of ASD-risk genes, indicating that disrupting the proper functioning of Pvalb neuron-containing neuronal networks by PVdownregulation is sufficient to induce an ASD phenotype in mice without another predisposition (Wöhr et al., 2015). In the last few years, we have tested different and well-established mouse models of ASD with a focus on Pvalb neurons. Unlike many other groups, we use two different markers to identify and quantify the number of Pvalb neurons: first, with an antibody against PV and second, biotinylated Vicia Villosa Agglutinin (WA) to label perineuronal nets (PNN), which consist of extracellular matrix components and preferentially surround Pvalb neurons, thus representing a second independent marker for identifying Pvalb neurons (Figure 1). Using this approach, we have shown that in postnatal day (PND) 25 PV-/- mice, Pvalb neurons are not absent-as could be deduced by the complete absence of PV immunoreactivity-but deficient of PV. Similar observations were made in PV+/-, Shank1-/-, Shank3B-/- (Filice, Vörckel, Sungur, Wöhr, \& Schwaller, 2016), and in utero valproic acid (VPA)-exposed mice (Lauber, Filice, \& Schwaller, 2016). In all of these models, we observe lower numbers of PV-immunoreactive cells and decreased PV protein (and Pvalb mRNA) levels compared to WT mice; however the number of VVA-positive PNN-ensheathed cells is unchanged, indicating that PV protein expression is strongly downregulated in Pvalb neurons of these genetic and environmental ASD mouse models (Figure 1). In support, RNA-seq and qRT-PCR analyses of post-mortem samples of frontal cortex, temporal cortex, and cerebellum from ASD individuals, compared to control individuals, revealed that the most significant changes include a decrease in PVALB mRNA levels (Parikshak et al., 2016). Accordingly, we hypothesize that Pvalb neurons undergo adaptive/homeostatic changes during early postnatal development, under pre-stressed conditions, such as the ones caused by mutations in proteins associated with synapses (e.g., SHANK proteins), or when challenged prenatally by environmental insults, as observed in the VPA model. Down-regulation of PV levels might enable a Pvalb neuron to modulate its own output and subsequently keep the excitation/inhibition balance of brain circuits within a proper "physiological" window during the maturation period of Pvalb neurons (i.e., mostly during the first postnatal month in mice). In this context, the ASD phenotype emerging in the $\mathrm{PV}-$ / - and other ASD mouse models with confirmed PV deficits may be viewed as the consequence or "cost" of such a correction.

Interestingly, in the VPA mouse model, PV down-regulation is restricted to the striatum, whereas no changes are found in the investigated cortical regions (medial prefrontal cortex, somatosensory cortex). However, in the cortex of PND25 VPA-exposed mice, we detect decreased levels of $\mathrm{K}_{\mathrm{v}} 3.1$, a voltage-dependent potassium channel that is exclusively expressed on the surface of Pvalb neurons. Blocking $\mathrm{K}_{\mathrm{v}} 3.1$ conductances or knocking out $K_{c n} 1$, the gene coding for $\mathrm{K}_{\mathrm{v}} 3.1$, leads to a broadening of AP duration due to a reduced rate of repolarization (Espinosa, Torres-Vega, Marks, \& Joho, 2008). As a result, neurotransmitter release from these neurons is increased, presumably enhancing Pvalb neuron-mediated inhibition. A similar effect is observed in PV-deficient Pvalb neurons, since the absence of PV increases short-term facilitation, which in turn leads to an enhanced 

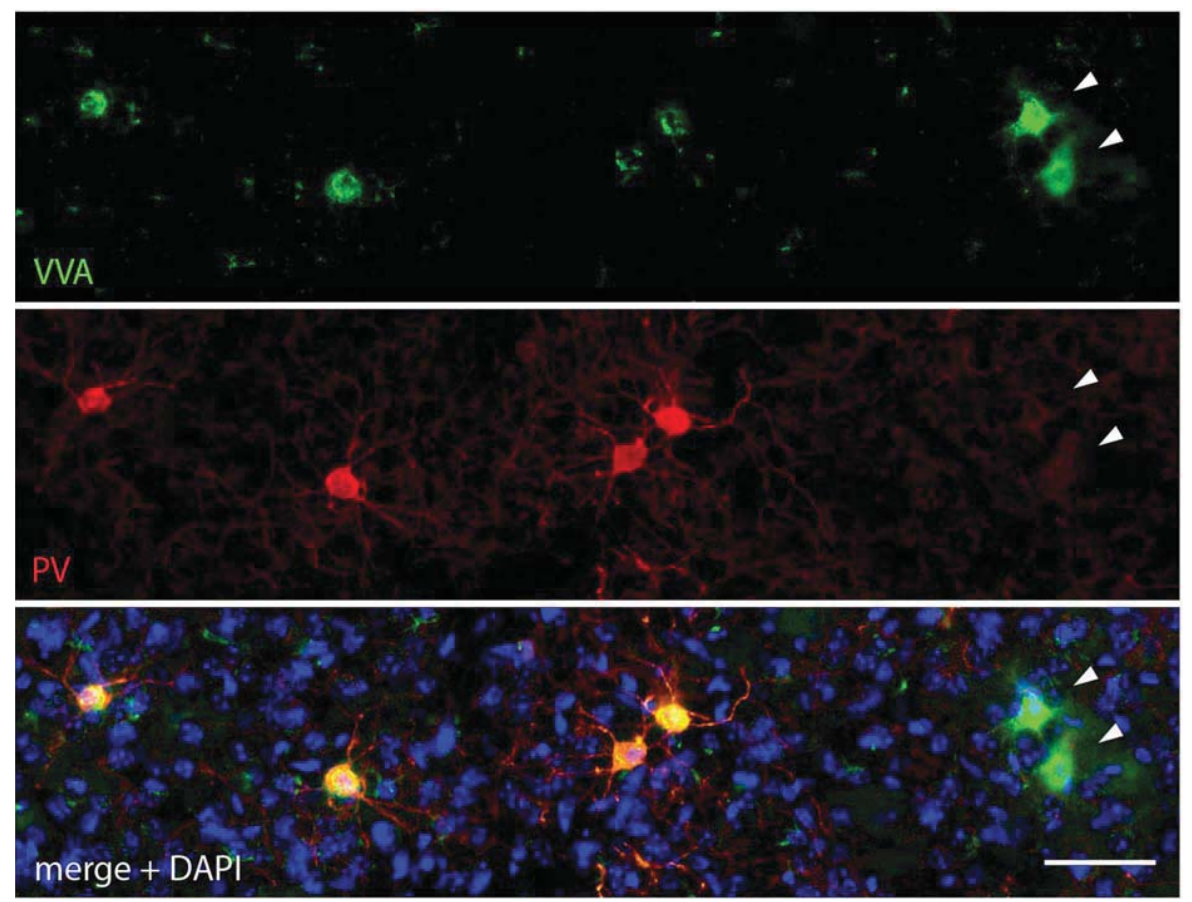

FIGURE 1 Section from the striatum of a VPA mouse. Pvalb neurons are visualized by VVA-positive PNN (green) and by antibodies against PV (red). The two cells on the right side (indicated by arrowheads) are presumably Pvalb neurons with low-to-absent PV levels. In the third panel, green and red images are merged and additionally cell nuclei are visualized by DAPI staining (blue). Scale bar: $50 \mu \mathrm{m}$

frequency-dependent output from Pvalb neurons (Schwaller, 2012). These observations indicate that the output of Pvalb neurons may be modulated by different means in a circuit-specific manner. Hence, reestablishing PV levels or modulating Pvalb cell neuronal output during critical periods of neurodevelopment (e.g., during the early postnatal period coinciding with PV upregulation and maturation of the Pvalb neuron network) might reduce or even abolish the core symptoms of ASD. Experiments aimed at answering these questions are currently underway in our lab. From a clinical point of view, this strategy represents a new and putatively promising approach for the treatment of ASD individuals with different predispositions.

\section{ORCID}

Emanuel Lauber (iD) http://orcid.org/0000-0002-6588-0374

Emanuel Lauber (D), Federica Filice, Beat Schwaller Anatomy Unit, Department of Medicine, University of Fribourg, Fribourg, Switzerland

Correspondence Emanuel Lauber, Anatomy Unit, Department of Medicine, University of Fribourg, Route Albert-Gockel 1, CH-1700 Fribourg, Switzerland. Email: emanuel.lauber@unifr.ch

Federica Filice, Anatomy Unit, Department of Medicine, University of Fribourg, Route Albert-Gockel 1, CH-1700 Fribourg, Switzerland. Email: federica.filice@unifr.ch

Beat Schwaller, Anatomy Unit, Department of Medicine, University of Fribourg, Route Albert-Gockel 1, CH-1700 Fribourg, Switzerland. Email: beat.schwaller@unifr.ch

\section{REFERENCES}

de la Torre-Ubieta, L., Won, H., Stein, J. L., \& Geschwind, D. H. (2016). Advancing the understanding of autism disease mechanisms through genetics. Nature Medicine, 22(4):345-361.

Johnson, M.H. (2017). Autism as an adaptive common variant pathway for human brain development. Developmental Cognitive Neuroscience, 25:5-11.

Hashemi, E., Ariza, J., Rogers, H., Noctor, S. C., \& Martínez-Cerdeño, V. (2017). The number of parvalbumin-expressing interneurons is decreased in the medial prefrontal cortex in autism. Cerebral Cortex, 27(3):1931-1943.

Wöhr, M., Orduz, D., Gregory, P., Moreno, H., Khan, U., Vörckel, K. J., . Schwaller, B. (2015). Lack of parvalbumin in mice leads to behavioral deficits relevant to all human autism core symptoms and related neural morphofunctional abnormalities. Translational Psychiatry, 5:e525.

Filice, F., Vörckel, K. J., Sungur, A. Ö., Wöhr, M., \& Schwaller, B. (2016). Reduction in parvalbumin expression not loss of the parvalbuminexpressing GABA interneuron subpopulation in genetic parvalbumin and shank mouse models of autism. Molecular Brain, 9:10.

Lauber, E., Filice, \& Schwaller. (2016). Prenatal valproate exposure differentially affects parvalbumin-expressing neurons and related circuits in the cortex and striatum of mice. Frontiers in Molecular Neuroscience, 9:150.

Parikshak, N. N., Swarup, V., Belgard, T. G., Irimia, M., Ramaswami, G., Gandal, M. J., ... Geschwind, D. H. (2016). Genome-wide changes in IncRNA, splicing, and regional gene expression patterns in autism. Nature, 540(7633):423-427.

Espinosa, Torres-Vega, Marks, \& Joho. (2008). Ablation of Kv3.1 and Kv3.3 potassium channels disrupts thalamocortical oscillations in vitro and in vivo. Journal of Neuroscience, 28(21):5570-5581.

Schwaller, B. (2012) The use of transgenic mouse models to reveal the functions of $\mathrm{Ca} 2+$ buffer proteins in excitable cells. Biochimica et Biophysica Acta, 1820(8):1294-1303. 